\title{
Leveraging compressive stresses to attenuate the electrical resistivity of
}

\section{buckypaper}

\author{
Vijay Kumar $^{\mathrm{a}}$, Henrik Haspel ${ }^{\mathrm{b}, \mathrm{c}}$, Krisztina Nagy ${ }^{\mathrm{b}, \mathrm{c}}$, Amit Rawal ${ }^{\mathrm{d}}$, Akos Kukovecz ${ }^{\mathrm{b}, \mathrm{c}^{*}}$ \\ ${ }^{a}$ University of Borås, SE-501 90 Borås, Sweden \\ ${ }^{b}$ MTA-SZTE “Lendület” Porous Nanocomposites Research Group, Rerrich Béla tér 1., Szeged, \\ Hungary \\ ${ }^{c}$ Department of Applied and Environmental Chemistry, University of Szeged, Rerrich Béla tér 1., \\ Szeged, Hungary \\ ${ }^{d}$ Department of Textile Technology, Indian Institute of Technology Delhi, Hauz Khas, New Delhi, \\ India
}

\begin{abstract}
Buckypaper (BP) is a planar film that consists of random network of multiwall carbon nanotubes (MWCNTs) held together by weak van der Waals interactions at tube-tube junctions. Although individual carbon nanotubes (CNTs) possess remarkable electrical properties, the electrical resistance of pristine BP is usually too high for practical applications. However, the electrical resistivity of BP can be attenuated by applying modest compressive stresses. Herein, we report an analytical model for predicting the electrical resistivity of BP under defined level of compressive strain. The predictive piezoresistive model of BP was developed by formulating a direct relationship with the structural parameters, physical and electrical properties of CNTs. The basis of the piezoresistive model relied upon the geometrical probability approach in combination with classical Hertzian contact mechanics and constriction resistance techniques. A comparison has been made between the theoretical and experimental results of electrical resistivity of BPs with varying densities. A reasonably good quantitative agreement was obtained between the theory and
\end{abstract}

\footnotetext{
*Corresponding Authors: E-mail: kakos@chem.u-szeged.hu (Akos Kukovecz), arawal@iitd.ac.in (Amit Rawal)
} 
experiments. The main source of error is caused by the uncertainty in the measurement of the initial BP thickness. Through theoretical modeling, initial fiber volume fraction was found to be one of key parameters that can modulate the piezoresistive behavior of BP.

\section{Introduction}

Carbon nanotubes (CNTs) exhibit remarkable physical, mechanical, thermal, and electrical properties. CNTs are usually synthesized in the form of vertically aligned [1] or random networks of fibers [2-5]. Aligned networks of CNTs have been found to be valuable in numerous applications but there are experimental challenges involved in the preparation $[5,6]$. It is significantly easier to prepare buckypaper (BP), which is a self-supporting random network of CNTs entangled together by means of weak van der Waals interactions at tube-tube junctions. Typically, BP is prepared via filtration of CNT suspensions resulting in a mesoporous material made up of layers of multiwall carbon nanotubes (MWCNTs) [4,7,8]. BP possesses mechanical and electrical properties that are attractive for numerous practical applications $[9,10]$. The favorable structural arrangement of CNTs and the abundance of tube-tube contacts in BP have led to an ever-increasing list of applications ranging from actuators [11], artificial muscles [12], strain sensors [13] to supercapacitors [14]. Despite the fact that an individual carbon nanotube has excellent electrical properties, electron transport from one nanotube to another is the bottleneck governing the overall electrical conductivity of BP [15]. Ostensibly, the contact resistance between the CNTs can be in the range of 0.1-1 M $\Omega$ [16]. One of the ways to enhance the electrical properties of BP can be envisaged through covalently bonded cross-linking of CNTs [17-20]. However, Zhang et al. [21] pointed out two critical issues in this approach. Firstly, chemical functionalization and electron beam irradiation can damage the intrinsic structure of the CNTs and secondly, the aggregation of CNTs can occur during the filtration of CNT suspensions. Alternatively, the contact resistance can be tuned by the orientation of CNTs and also through the application of modest stresses at the contacts 
[22]. Since the latter method is the simplest and least resource-demanding, it is of imperative to predict the piezoresistive behavior of BP by formulating a relationship between the structural characteristics, constituent CNT dimensions and the electrical properties.

In the past, the porous and random nature of CNT network in BP has been compared to that of a nonwoven textile material with randomly oriented fibers [23]. Initial efforts were focused on understanding the compression resistance of BP $[24,25]$ through the classical semi-empirical model of van Wyk [26]. The analytical models of BP compressibility were also developed by formulating the relationship between orientation distribution, CNT volume fraction along with physical and mechanical properties $[27,28]$. Recently, the compression model of BP together with constriction resistance techniques has been used to predict compression induced electrical response [29]. However, compressive stress is a pre-requisite for predicting the electrical response, which requires additional experimental work. Thus, the main aim of the present research is to predict the electrical resistivity of BP under defined levels of compressive strain by formulating a direct relationship with structural parameters, physical and electrical properties of CNTs. Here, a simple twodimensional (2D) piezoresistive model of BP is developed through a multi-stage process. Initially, the structural parameters of a planar BP have been computed through geometrical probability approach followed by computation of compressive stresses. Subsequently, the classical Hertzian contact mechanics approach in combination with constriction resistance techniques led to the predictive model of electrical resistivity of BP. Further, a comparison has been made between the theoretical and experimental results of electrical resistivity of BPs with varying densities. A parametric analysis has also been performed to understand the effect of key structural parameters on electrical resistivity of BP. 


\section{Theoretical Analysis}

The following assumptions have been made in order to develop a predictive model of electrical resistivity of BP.

- CNTs in BP have identical properties in terms of physical dimensions (diameter, length) and exhibit linearly elastic characteristics.

- CNT segments between the junctions are straight before loading. Nevertheless, the geometrical probability approach accounts for the local waviness in CNTs by considering the changes in the orientation distribution of CNTs through tangents drawn at the wavy line [30].

- Slippage between the CNTs in the network is negligible and other deformation types (torsion, compression and tensile) of CNTs are considered to be small.

- The internal electrical resistance of CNT is low enough [16] to be neglected in the analysis.

\subsection{Structural parameters of $B P$}

As mentioned earlier, BP consists of a network of CNTs. The spatial alignment of each CNT can be represented by a pair of in-plane $(\varphi)$ and out-of-plane $(\theta)$ orientation angles in a spherical coordinate system. Accordingly, the probability that the CNT direction lies in an infinitesimal range of $\theta$ and $\theta+d \theta$, and $\varphi$ and $\varphi+d \varphi$ is $\Omega(\theta, \varphi) \sin \theta d \theta d \varphi$, where $\Omega(\theta, \varphi)$ is the orientation distribution function (ODF). The ODF is abided by the following normalization condition.

$$
\int_{0}^{\pi} d \varphi \int_{0}^{\pi} \sin \theta \Omega(\theta, \varphi) d \theta=1
$$

In the past, the MWCNTs in BP were experimentally stratified as randomly oriented entities [4,5,27]. Accordingly, the geometrical probability approach, as proposed by Komori and 
Makishima [30] for fibrous assemblies has been applied for computing the structural parameters of BP. Here, an individual CNT can be considered analogous to a fiber in Komori and Makishima's model [30], hence the total number of CNT-CNT contacts per unit volume $\left(n_{v}\right)$ and the mean distance between the CNT-CNT contacts $(\bar{b})$ inside a BP can be computed as shown below [30],

$$
n_{v}=\frac{16 I \phi^{2}}{\pi^{2} d_{o}^{3}} \quad \text { and } \bar{b}=\frac{\pi d_{o}}{8 \phi I}
$$

where $I=\int_{0}^{\pi} d \theta \int_{0}^{\pi} J(\theta, \varphi) \sin \theta \Omega(\theta, \varphi) d \varphi ; J(\theta, \varphi)=\int_{0}^{\pi} d \varphi^{\prime} \int_{0}^{\pi} d \theta^{\prime} \Omega\left(\theta^{\prime}, \varphi^{\prime}\right) \sin \chi\left(\theta, \varphi, \theta^{\prime}, \varphi^{\prime}\right) \sin \theta^{\prime}$ and $\sin \chi\left(\theta, \varphi, \theta^{\prime}, \varphi^{\prime}\right)=\left[1-\left\{\cos \theta \cos \theta^{\prime}+\sin \theta \sin \theta^{\prime} \cos \left(\varphi-\varphi^{\prime}\right)^{2}\right\}\right]^{1 / 2}$

where $\phi$ is the volume fraction of CNT, $d_{o}$ is the outer diameter of MWCNT, $I$ is an orientation parameter defining the alignment of CNTs in the assembly, and $\mathcal{X}$ is the angle between the two axes of CNTs having defined types of orientation distributions, $\Omega(\theta, \varphi)$ and $\Omega\left(\theta^{\prime}, \varphi^{\prime}\right)$.

In the past, the morphology of BP has been found to be analogous to that of two-dimensional (2D) paper-like structure [31]. Therefore, in the present work, the BP has been considered as a layered 2D network of CNTs stacked over each other such that the number of tubes are same as that of real three-dimensional (3D) structure [32]. Since, the out-of-plane orientation angle in a 3D structure is required to be updated under defined level of compressive strain, which tends to be computationally intensive. Assuming all the CNTs lie in the in-plane direction such that $\theta=\theta^{\prime}=\frac{\pi}{2} \quad$ and $\quad$ using $\quad$ Dirac's delta function, $\quad \Omega(\theta, \varphi)=\Omega(\varphi) \cdot \delta(\pi / 2-\theta) \quad$ and $\Omega\left(\theta^{\prime}, \varphi^{\prime}\right)=\Omega\left(\varphi^{\prime}\right) . \delta\left(\pi / 2-\theta^{\prime}\right)$. Based upon these considerations, equation (3) becomes, 


$$
\begin{aligned}
& \sin \chi\left(\pi / 2, \varphi, \pi / 2, \varphi^{\prime}\right)=\left|\sin \left(\varphi-\varphi^{\prime}\right)\right|, J(\pi / 2, \varphi)=\int_{0}^{\pi} d \varphi^{\prime} \Omega\left(\varphi^{\prime}\right)\left|\sin \left(\varphi-\varphi^{\prime}\right)\right| \text { and } \\
& I=\int_{0}^{\pi} d \varphi \int_{0}^{\pi} d \varphi^{\prime} \Omega(\varphi) \Omega\left(\varphi^{\prime}\right)\left|\sin \left(\varphi-\varphi^{\prime}\right)\right|
\end{aligned}
$$

The above expression of $I$ matches well with the orientation function $(f)$ as defined by Toll and Manson [33]. For a 2D random network, $\Omega(\varphi)=\Omega\left(\varphi^{\prime}\right)=\Omega_{0}=\frac{1}{\pi}$ and $I=\frac{2}{\pi}$.

\subsection{Compression deformation of $B P$}

As aforementioned, a BP is a network of CNTs held together by weak van der Waals forces acting at CNT-CNT contacts. During compression loading, the role of van der Waals forces at CNT-CNT contact is negligible [24], although this may not hold true during unloading. In this analysis, we have accordingly neglected the role of van der Waals forces during the application of compressive stresses. When the BP is subjected to uniaxial compression, the applied compressive stresses are transmitted within the network by means of layers of CNT-CNT contacts [24,27-29], as illustrated in Fig. 1.

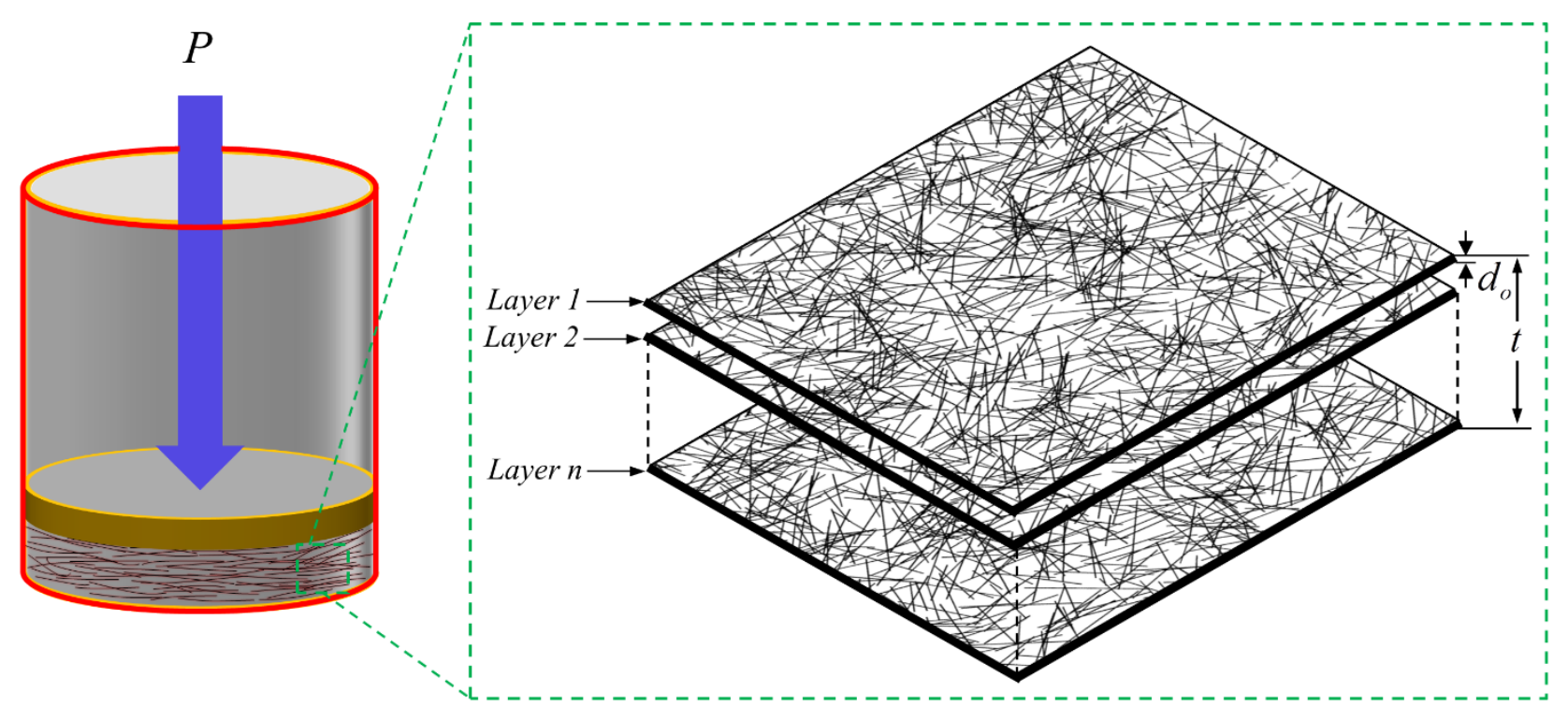

Fig. 1. A schematic depicting the uniaxial compression of BP and the magnified view illustrates 
the transfer of compression load within the layers of CNTs. Here, $P$ is the compressive stress applied at BP, $t$ is the thickness of BP, and $d_{o}$ is the outer diameter of MWCNT.

Each layer of CNT-CNT contact is assumed to have a thickness equivalent to that of the outer diameter of CNT $\left(d_{o}\right)$. Since, the CNTs are distributed in a stochastic manner, the applied stress is distributed statistically [33]. Thus, the mean force experienced by the CNT-CNT contact $(\bar{p})$ as a function of applied compressive stress $(P)$ is given below,

$$
\bar{p}=\frac{P}{n_{v} d_{o}}
$$

Now considering a segment of CNT between the two successive contacts as a representative volume element (RVE) of the BP. Based upon the principles of continuum mechanics, the strain experienced by the RVE due to the compressive stresses would emulate the deformation occuring at the macroscale. Hence, the mean deflection of the network can be calculated by assuming the CNT segment as a beam with as shown below [34],

$$
\bar{\delta}=\frac{\bar{b}^{3}}{3 E_{C N T} I_{C N T}} \bar{p}
$$

where

$$
I_{C N T}=\frac{\pi\left(d_{o}^{4}-d_{i}^{4}\right)}{64} \text { for MWCNT }
$$

where $E_{C N T}$ is the Young's modulus of the CNT, $I_{C N T}$ is the moment of inertia of CNT and $d_{i}$ is the inner diameter of MWCNT.

Combining equations (5) and (6) and differentiating the resultant equation w.r.t. P, i.e.

$$
d \bar{\delta}=\frac{\bar{b}^{3}}{3 E_{C N T} I_{C N T} n_{\nu} d_{o}} d P
$$


According to Toll and Manson [33], the network is collapsed when $\bar{\delta}=d_{o}$ such that the total volume of the network becomes zero. Assuming $\bar{\delta}<<d_{o}$, a simple relationship between $d \bar{\delta}$ and $\phi$ has been formulated [33]:

$$
d \bar{\delta}=d_{o} \frac{d \phi}{\phi}
$$

Hence, combining Eqs. (2), (7)-(9),

$$
d P=\frac{384 E_{C N T} I^{4}}{\pi^{4}}\left(1-\frac{d_{i}^{4}}{d_{o}^{4}}\right) \phi^{4} d \phi
$$

The total stress required to compress the BP from initial volume fraction of CNT $\left(\phi_{o}\right)$ to a defined volume fraction $(\phi)$ can be calculated as

$$
P=\int_{0}^{P} d P=\int_{\phi_{0}}^{\phi} \frac{384 E_{C N T} I^{4}}{\pi^{4}}\left(1-\frac{d_{i}^{4}}{d_{o}^{4}}\right) \phi^{4} d \phi
$$

Thus,

$$
P=\frac{384 E_{C N T} I^{4}}{5 \pi^{4}}\left(1-\frac{d_{i}^{4}}{d_{o}^{4}}\right)\left(\phi^{5}-\phi_{0}^{5}\right)
$$

Interestingly, $\phi$ and $\phi_{o}$ are related with each other through the following equation [27].

$$
\phi=\frac{\phi_{0}}{1-\varepsilon}
$$

The applied compressive stress in the BP not only bends the CNT element but also causes an elliptical contact region due to transverse compression [29]. These elliptical shaped contact regions forms "a" spots in order to channelize the flow of electrons [35]. For two CNTs with their axes crossing at an angle $\chi$, the inter-CNT contact area is given by [36], 


$$
A_{c}=\pi m n\left\{\frac{3 p d_{o}\left(1-v^{2}\right)}{4 E_{C N T}}\right\}^{2 / 3}
$$

where $p$ is the force acting on CNT-CNT contact, $v$ is the Poisson's ratio of the CNT, $m$ and $n$ are the transcendental functions of crossing angle $(\chi)$ or $\left(\varphi-\varphi^{\prime}\right)$ in $2 \mathrm{D}$. The values of $m$ and $n$ for a given $\chi$ can be obtained from Ref. [36].

Since BP consists of a network of stochastically distributed CNTs, the orientation averaging approach has been applied for computing the mean contact area, $\bar{A}_{c}$ [29]. Accordingly, $p$ needs to be appropriately replaced by $\bar{p}$ using equation (14). Similarly, the mean crossing angle of CNTs, $\langle\chi\rangle$ based upon simple orientation averaging scheme would replace $\chi$ in equation (14). Thus,

$$
\bar{A}_{c}=\pi m n\left\{\frac{3 \bar{p} d_{o}\left(1-v^{2}\right)}{4 E_{C N T}}\right\}^{2 / 3}
$$

\subsection{Electrical Resistivity of $B P$}

In general, the electron transport in CNT assembly encounters two types of hindrances: internal resistance of individual CNT and constriction resistance [16,29]. The internal resistance $\left(r_{i}\right)$ is caused by the inherent electrical resistance of the nanotube itself, whereas the constriction or contact resistance $\left(r_{c}\right)$ originates from the contact area formed between the two contacting tubes and typically, $r_{c}>>r_{i}$ for CNTs [16]. An elliptical contact region is formed between two CNTs and accordingly, $r_{c}$ can be calculated using following equation [35],

$$
r_{c}=\frac{\rho}{4 a_{c}} f(\sqrt{\eta})
$$

where $\eta=\frac{m}{n}, a_{c}$ is the radius of a circular spot with the area identical to that of the elliptical aspot, $\rho$ is the electrical resistivity, and $f(\sqrt{\eta})$ is the form factor function. 
Due to the stochastic nature of CNTs in BP, $a_{c}$ can be replaced by $\bar{a}_{c}$ in equation (16) to obtain the mean contact resistance,

$$
\bar{r}_{c}=\frac{\rho}{4 \bar{a}_{c}} f(\sqrt{\bar{\eta}})
$$

where $\bar{a}_{c}=\left(\frac{\bar{A}_{c}}{\pi}\right)^{1 / 2}$ and $\bar{\eta}$ is the ratio of mean of semi-major to semi-minor axes of the constriction which effectively is a function of $\langle\chi\rangle$.

As mentioned earlier, BP consists of stacks of CNT layers, each layer has a thickness of $d_{o}$ and the resistance of each layer can be considered as a network of parallel resistors [16]. Thus, the equivalent electrical resistance of the $\mathrm{BP}(R)$ is given by,

$$
R=\bar{r}_{c} \frac{n_{l}}{n_{c l}}
$$

where $n_{l}=\frac{t}{d_{o}}$ and $n_{c l}=n_{v} A d_{o}=\frac{16 I \phi^{2}}{\pi^{2} d_{o}^{2}} A$

where $n_{l}$ is the number of layers, $t$ is the BP thickness, $A$ is the surface area of the BP and $n_{c l}$ is the number of CNT-CNT contacts in each layer.

Furthermore, the resistivity of the $\mathrm{BP}\left(\rho_{s}\right)$ can be calculated using the following equation,

$$
\rho_{s}=\frac{R A}{t}
$$

Combining equations (17)-(19),

$$
\rho_{s}=\frac{\pi^{2} d_{o}}{16 I \phi^{2}} \frac{\rho}{4 \bar{a}_{c}} f(\sqrt{\bar{\eta}})
$$


Thus, the electrical resistivity of BP can be predicted through equations (4), (5), (12), (13) and (15). A Matlab ${ }^{\circledR}$ code was written for predicting the electrical resistivity of BP under a defined level of compressive strain using an iterative process.

\section{Experimental Work}

Multiwall carbon nanotubes (MWCNTs) were synthesized by 2 hours of catalytic chemical vapor deposition from a $\mathrm{C}_{2} \mathrm{H}_{4}: \mathrm{N}_{2}\left(30: 300 \mathrm{~cm}^{3} / \mathrm{min}\right)$ gas mixture at $650{ }^{\circ} \mathrm{C}$ over $\mathrm{Fe}, \mathrm{Co} / \mathrm{Al}_{2} \mathrm{O}_{3}(2.5-2.5$ wt.\% metal loading) catalyst. The MWCNTs were purified by repeating 4 hours of refluxing in 10 $\mathrm{mol} \cdot \mathrm{dm}^{-3}$ aqueous $\mathrm{NaOH}$ then 4 hours in cc. $\mathrm{HCl}$ solution four times. We refer to Ref. [37] for more details about the synthesis and purification procedure. Individual nanotubes were characterized using a FEI Tecnai G2 $200 \mathrm{kV}$ transmission electron microscope (TEM). Nanotubes were converted into BP by filtering $150 \mathrm{~cm}^{3}$ of their $0.1 \mathrm{~g} \cdot \mathrm{dm}^{-3} \mathrm{~N}, \mathrm{~N}$-dimethylformamide suspensions through a $15 \mathrm{~mm}$ Sartolon polyamide membrane filter with nominal pore diameter of $0.45 \mu \mathrm{m}$. The BPs were dried at $80{ }^{\circ} \mathrm{C}$ overnight in air on the filters and peeled off in acetone afterwards [38]. Three different sets of BP samples with varying densities were prepared and the properties are given in Table 1.

The absolute density of the as-prepared CNTs was measured with a Micromeritics MultiVolume Pycnometer 1305 using helium as pressurizing gas. The morphology of the surface and the crosssection of the BPs were characterized using a Hitachi S-4700 Type II field-emission scanning electron microscope (SEM). Typical transmission electron microscopy (TEM) and scanning electron microscopy (SEM) images of the individual nanotubes and the formed BPs are presented in Fig. 2. SEM images were quantitatively analyzed using the ImageJ ${ }^{\circledR}$ software. Measurements of 100 nanotubes taken in 3 different images were averaged to get the length and diameter values reported in Table 1. 




Fig. 2. Characteristic TEM (a, b) and SEM (c, d) images of the BP used in this study. Part c shows the buckypaper from the top (BP is normal to the direction of filtration). Filtration direction in part $\mathrm{d}$ is along the top left - bottom right diagonal of the image.

Table 1 Properties of BPs and its constituents

\begin{tabular}{|c|c|c|c|c|c|}
\hline Parameter & Units & BP1 & BP2 & BP3 & Source \\
\hline Initial density of BP $\left(m_{\rho B P}\right)$ & $g . \mathrm{cm}^{-3}$ & 0.072 & 0.082 & 0.089 & Measured \\
\hline Initial volume fraction of CNTs $\left(\phi_{0}\right)$ & - & 0.025 & 0.028 & 0.031 & Calculated \\
\hline Outer diameter of CNT $\left(d_{o}\right)$ & $\mathrm{nm}$ & \multicolumn{3}{|c|}{$14.1 \pm 7.5$} & Measured \\
\hline Inner diameter of CNT $\left(d_{i}\right)$ & $\mathrm{nm}$ & \multicolumn{3}{|c|}{$5.8 \pm 1.7$} & Measured \\
\hline Length of CNT $(l)$ & $\mu m$ & \multicolumn{3}{|c|}{$23.94 \pm 3.64$} & Measured \\
\hline Mass density of CNT $\left(m_{\rho C N T}\right)$ & $g \cdot \mathrm{cm}^{-3}$ & $\Omega .88$ & {$[15]$} \\
\hline Electrical resistivity of CNT $(\rho)$ & $\Omega .68 \times 10^{-5}$ & \\
\hline
\end{tabular}


BP compression experiments were performed in a purpose-built cell that allowed the compression of the carbon nanotube mat in $16.5 \mu \mathrm{m}$ units. The measurement apparatus is depicted in Fig. 3. BP samples were placed between two copper disk electrodes (diameter $=15 \mathrm{~mm}$, height $=2 \mathrm{~mm})$, and their thickness was measured at every applied pressure with a micrometer screw with $1 \mu \mathrm{m}$ precision (Moore \& Wright Electronic Outside Micrometer). An in-situ two-point electrical resistivity measurement technique was applied to follow the resistivity change of the compressed films. During compression loading, the electrical resistance was recorded using a Voltcraft VC920 digital multimeter operated at constant current mode. The measuring current was approx. $1.1 \mathrm{~mA}$. The corresponding electrical resistivity of BPs under defined compressive strain was then calculated from the geometry of the film at each measurement step. Each resistance step was measured 15 minutes after compressing the BP film by 1 unit to allow for the decay of any transient effects. Electrical resistance and film thickness data were normalized to the initial resistance and thickness. Experiments were performed on three individual freshly filtered specimens (denoted as S1, S2, S3) for each BP sample with varying density. 


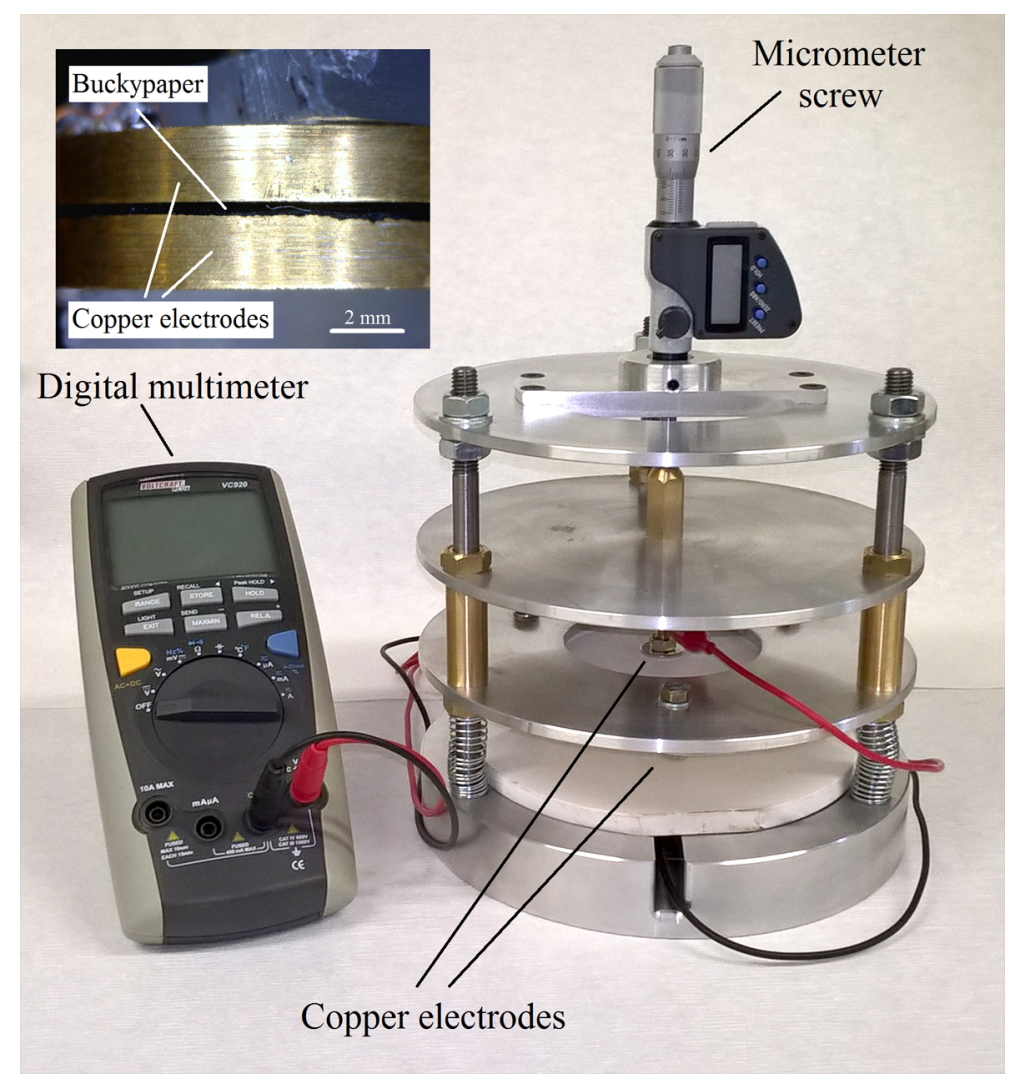

Fig. 3. A photo of the experimental setup used for determining electrical resistivity of BP under defined compressive strain. The insert shows a close-up side view of the BP mounted between the two electrode disks.

\section{Results and Discussion}

Compression of BP inevitably involves boundary effects resulting in "barrelling" or "hyperboloid" deformations [27]. In the past, these effects have led to significant differences between theory and experiments [27]. Despite the fact that such effects have not been accounted in our theoretical analysis, a reasonably good agreement was obtained between the theoretical and experimental results of electrical resistivity of BPs with varying densities as shown in Fig. 4. Here, three specimens were tested for each sample of BP and the behavior of each sample is clearly marked in Fig. 4. 
(a)

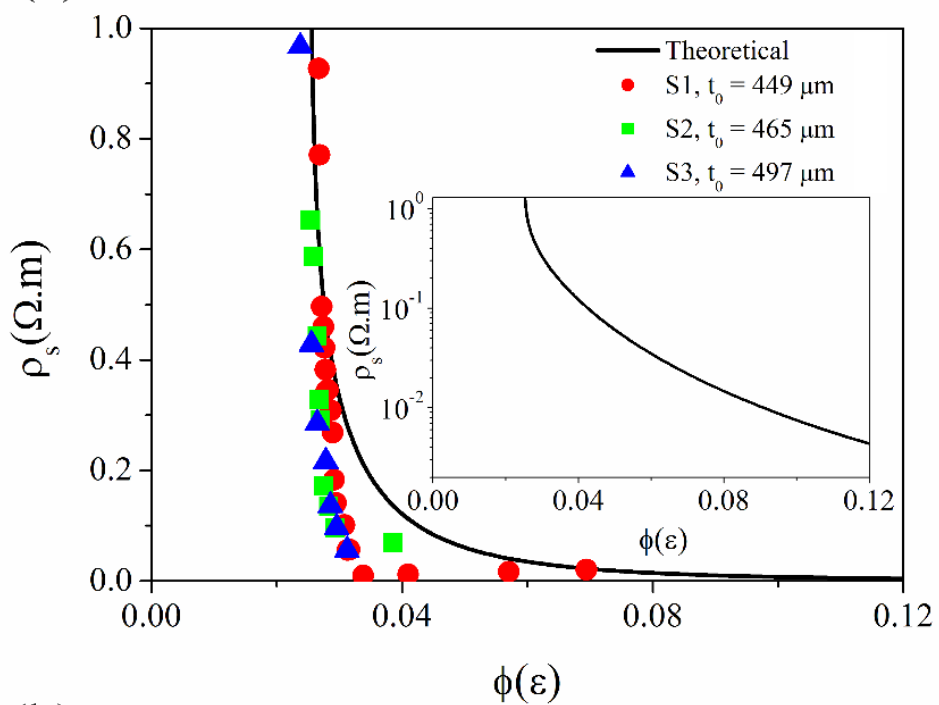

(b)

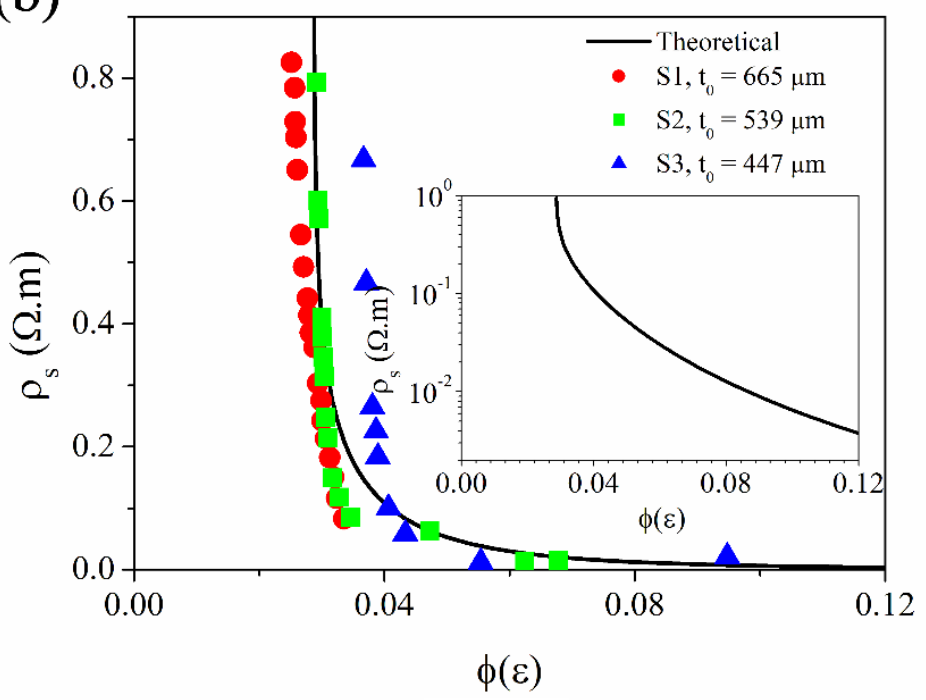

(c)

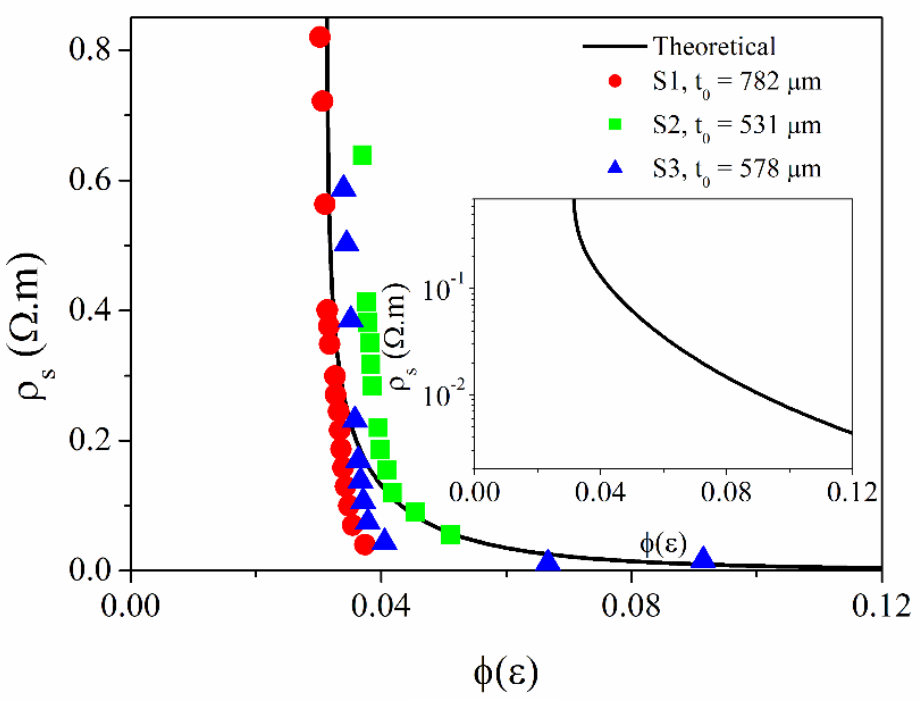


Fig. 4. Comparison between theoretical and experimental results of compression induced electrical resistivity of (a) BP1 (b) BP2 and (c) BP3. Here, S1, S2 and S3 represent three individual specimens that have been used for determining the electrical resistivity of each BP sample and $t_{0}$ is the initial thickness of BP.

One of the possible reasons for the discrepancy between the theoretical and experimental results is the difficulties encountered during the measurement of BP thickness under zero compressive stress [27]. This is particularly pertinent for the BP samples with lower densities, for example, sample BP1 that has the lowest density and initial volume fraction of CNTs. The experimental observations clearly demonstrate that there is a sharp decrease in electrical resistivity when such a sample is compressed gradually due to the significant reduction in BP thickness. Theoretically, there is a steady decrease in electrical resistivity during compression as reduction in thickness has been carried out in a fairly homogenous way using equation (13).

Next, we propose the mechanism of piezoresistive behavior of BP similar to that of CNT aerogels [39]. During uniaxial compression of BP, the stresses are transmitted within the network via layers of CNT-CNT contacts. Each CNT experiences a compression load that tends to bend the nanotube. This inevitably allows the interaction with other CNTs through the formation of new contacts. These new contacts increase the overall contact area that reduces the electrical resistivity of BP. It should be noted that the realignment of CNTs under compression load has not been taken into account as the structure of $\mathrm{BP}$ is considered to be 2D in nature. However, a 3D network of CNTs in the form of aerogels or sponges involves realignment of out-of-plane orientations during the application of compressive stresses [28,39]. Nevertheless, the initial alignment and volume fractions of CNTs in BP are the key parameters that affect the compression induced electrical resistivity. The random initial alignment of carbon nanomaterials results in lower electrical resistivity due to the higher number of contacts formed between the entities in comparison to the preferentially aligned structure [29]. However, increasing the compressive strain reduces the 
differences between the magnitudes of electrical properties of random and preferentially aligned structures as similar levels of number of contacts are attained in both cases [29]. Alternatively, increasing the initial volume fraction of CNTs in BP is anticipated to reduce the electrical resistivity significantly. To assess the effect of initial volume fraction of CNTs on electrical resistivity of BP, a parametric analysis was carried out, as illustrated in Fig. 5.

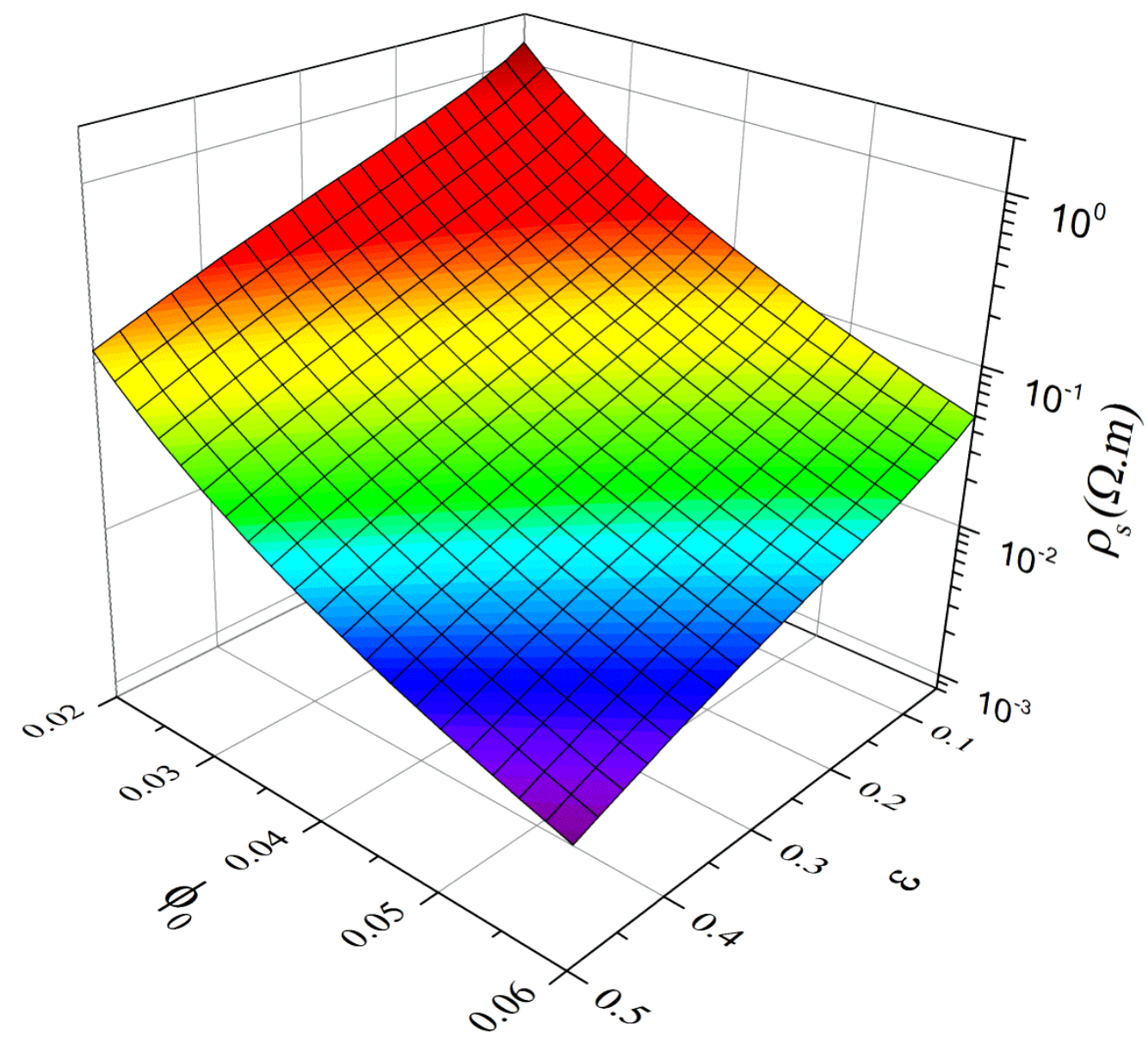

Fig. 5. Theoretical electrical resistivity of the BP $\left(\rho_{s}\right)$ as a function of initial volume fraction of $\operatorname{CNTs}\left(\phi_{0}\right)$ and compressive strain $(\varepsilon)$.

Interestingly, the electrical resistivity of BP has been reduced by nearly 27 times when a three-fold increase in the initial volume fraction $(\sim 0.02-0.06)$ of CNTs was carried out. Similar trends emerged when the compressive strain was increased from 5\% to 50\%. Given the potential of initial 
volume fraction of CNTs to reduce the electrical resistivity of $\mathrm{BP}$, it is imperative to know the maximum permissible volume fraction $\left(\phi_{\max }\right)$ for a 2D random assembly. Pan et al. [40] have formulated a relationship between $\phi_{\max }$ and the aspect ratio $(s)$ of constituent entities for a $2 \mathrm{D}$ random network, i.e. $\phi_{\max }<\frac{\pi^{2}}{16 \xi}$ where $\xi=\frac{1}{\pi} \ln \cot ^{2} \frac{\arcsin \left(\frac{1}{s}\right)}{2}$. However, it is notable that $\phi$ under defined level of $\varepsilon$ using equation (13) cannot theoretically exceed $\phi_{\max }$ of BP. For example, consider the aspect ratio of $\mathrm{CNT}$ as 1600 leading to $\phi_{\max }$ of $12 \%$ which can be attained with $\phi_{0}$ of $6 \%$ under $\varepsilon$ of $50 \%$. Thus, the threshold level of compressive strain for maximizing the volume fraction of CNTs in BP would inadvertently affect the electrical resistivity accordingly.

\section{Conclusions}

A simple 2D model for predicting the electrical resistivity of BP under defined levels of compressive strain has been proposed by formulating a direct relationship with the structural parameters, physical and electrical properties of CNTs. The stochastic nature of CNTs in BPs has been accounted using a geometrical probability approach that allowed the computation of structural parameters. Subsequently, the classical Hertzian contact mechanics and constriction resistance techniques were combined together for predicting the electrical resistivity of BP. The model of electrical resistivity under defined compressive strain was validated with BPs having varying densities. In general, a reasonably good agreement was observed between the theoretical and experimental results. The discrepancy between theory and experiments was attributed to the measurement of initial thickness under zero compressive stress. Through analytical modeling, key structural parameters of BPs have been clearly elucidated. Initial fiber volume fraction is one of the key parameters that affect the piezoresistive behavior of BP. The maximum volume fraction of CNTs evolved under the defined level of compressive strain can tune the electrical resistivity of 
BP. It is anticipated that these findings would help in designing of buckypaper-based sensors. One particularly interesting potential application area is the development of intelligent clothing, as buckypaper is highly compatible with other fibrous materials. Therefore, BP could act as a primary source relaying stress-, wear- or environment (humidity, temperature) data from textiles to the user and to the Internet of Things.

\section{Acknowledgement}

$\mathrm{HH}, \mathrm{KN}$ and $\mathrm{AK}$ acknowledge the financial support of the Hungarian National Research, Innovation and Development Office through projects NKFIH OTKA K 112531 and NN 110676.

\section{References}

[1] M. Chhowalla, K.B.K. Teo, C. Ducati, N.L. Rupesinghe, G.A.J. Amaratunga, A.C. Ferrari, D. Roy, J. Robertson, W.I. Milne, Growth process conditions of vertically aligned carbon nanotubes using plasma enhanced chemical vapor deposition, J. Appl. Phys. 90 (2001) $5308-5317$.

[2] A.G. Rinzler, J. Liu, H. Dai, P. Nikolaev, C.B. Huffman, F.J. Rodriguez-Macias, P.J. Boul, A.H. Lu, D. Heymann, D.T. Colbert, others, Large-scale purification of single-wall carbon nanotubes: process, product, and characterization, Appl. Phys. Mater. Sci. Process. 67 (1998) 29-37.

[3] M.S. Shaffer, X. Fan, A.H. Windle, Dispersion and packing of carbon nanotubes, Carbon. 36 (1998) 1603-1612.

[4] R.L. Whitby, T. Fukuda, T. Maekawa, S.L. James, S.V. Mikhalovsky, Geometric control and tuneable pore size distribution of buckypaper and buckydiscs, Carbon. 46 (2008) 949956. 
[5] R.L. Whitby, T. Fukuda, T. Maekawa, S.V. Mikhalovsky, A.B. Cundy, Real-time imaging of complex nanoscale mechanical responses of carbon nanotubes in highly compressible porous monoliths, Nanotechnology. 21 (2010) 75707.

[6] R.H. Poelma, X. Fan, Z.-Y. Hu, G. Van Tendeloo, H.W. van Zeijl, G.Q. Zhang, Effects of Nanostructure and Coating on the Mechanics of Carbon Nanotube Arrays, Adv. Funct. Mater. 26 (2016) 1233-1242.

[7] Z. Špitalskỳ, C. Aggelopoulos, G. Tsoukleri, C. Tsakiroglou, J. Parthenios, S. Georga, C. Krontiras, D. Tasis, K. Papagelis, C. Galiotis, The effect of oxidation treatment on the properties of multi-walled carbon nanotube thin films, Mater. Sci. Eng. B. 165 (2009) 135138.

[8] A. Ansón-Casaos, J.M. González-Domínguez, E. Terrado, M.T. Martínez, Surfactant-free assembling of functionalized single-walled carbon nanotube buckypapers, Carbon. 48 (2010) 1480-1488.

[9] G. Schuszter, E.-S. Bogya, D. Horváth, Á. Tóth, H. Haspel, Á. Kukovecz, Liquid droplet evaporation from buckypaper: On the fundamental properties of the evaporation profile, Microporous Mesoporous Mater. 209 (2015) 105-112.

[10] E. Mansfield, A. Feldman, A.N. Chiaramonti, J. Lehman, A.E. Curtin, Morphological and Electrical Characterization of MWCNT Papers and Pellets, J. Res. Natl. Inst. Stand. Technol. 120 (2015) 304.

[11] R.H. Baughman, C. Cui, A.A. Zakhidov, Z. Iqbal, J.N. Barisci, G.M. Spinks, G.G. Wallace, A. Mazzoldi, D. De Rossi, A.G. Rinzler, others, Carbon nanotube actuators, Science. 284 (1999) 1340-1344.

[12] U. Vohrer, I. Kolaric, M.H. Haque, S. Roth, U. Detlaff-Weglikowska, Carbon nanotube sheets for the use as artificial muscles, Carbon. 42 (2004) 1159-1164. 
[13] L. Camilli, C. Pisani, M. Passacantando, V. Grossi, M. Scarselli, P. Castrucci, M. De Crescenzi, Pressure-dependent electrical conductivity of freestanding three-dimensional carbon nanotube network, Appl. Phys. Lett. 102 (2013) 183117.

[14] S. Roy, R. Bajpai, N. Soin, P. Bajpai, K.S. Hazra, N. Kulshrestha, S.S. Roy, J.A. McLaughlin, D.S. Misra, Enhanced Field Emission and Improved Supercapacitor Obtained from Plasma-Modified Bucky Paper, Small. 7 (2011) 688-693.

[15] Q.W. Li, Y. Li, X.F. Zhang, S.B. Chikkannanavar, Y.H. Zhao, A.M. Dangelewicz, L.X. Zheng, S.K. Doorn, Q.X. Jia, D.E. Peterson, others, Structure-dependent electrical properties of carbon nanotube fibers, Adv. Mater. 19 (2007) 3358-3363.

[16] A. Allaoui, S.V. Hoa, P. Evesque, J. Bai, Electronic transport in carbon nanotube tangles under compression: The role of contact resistance, Scr. Mater. 61 (2009) 628-631.

[17] J. Zhang, D. Jiang, Interconnected multi-walled carbon nanotubes reinforced polymermatrix composites, Compos. Sci. Technol. 71 (2011) 466-470.

[18] A. Kis, G. Csanyi, J.-P. Salvetat, T.-N. Lee, E. Couteau, A.J. Kulik, W. Benoit, J. Brugger, L. Forro, Reinforcement of single-walled carbon nanotube bundles by intertube bridging, Nat. Mater. 3 (2004) 153-157.

[19] D.N. Ventura, R.A. Stone, K.-S. Chen, H.H. Hariri, K.A. Riddle, T.J. Fellers, C.S. Yun, G.F. Strouse, H.W. Kroto, S.F. Acquah, Assembly of cross-linked multi-walled carbon nanotube mats, Carbon. 48 (2010) 987-994.

[20] S.I. Cha, K.T. Kim, K.H. Lee, C.B. Mo, Y.J. Jeong, S.H. Hong, Mechanical and electrical properties of cross-linked carbon nanotubes, Carbon. 46 (2008) 482-488.

[21] J. Zhang, D. Jiang, H.-X. Peng, F. Qin, Enhanced mechanical and electrical properties of carbon nanotube buckypaper by in situ cross-linking, Carbon. 63 (2013) 125-132.

[22] A. Buldum, J.P. Lu, Contact resistance between carbon nanotubes, Phys. Rev. B. 63 (2001) 161403. 
[23] M.A. Atwater, A.K. Mousavi, Z.C. Leseman, J. Phillips, Direct synthesis and characterization of a nonwoven structure comprised of carbon nanofibers, Carbon. 57 (2013) 363-370.

[24] A. Allaoui, S. Toll, P. Evesque, J. Bai, On the compressive response of carbon nanotube tangles, Phys. Lett. A. 373 (2009) 3169-3173.

[25] A.K. Mousavi, M.A. Atwater, B.K. Mousavi, M. Jalalpour, M.R. Taha, Z.C. Leseman, Mechanical and electrical characterization of entangled networks of carbon nanofibers, Materials. 7 (2014) 4845-4853.

[26] C.M. Van Wyk, 20-Note on the compressibility of wool, J. Text. Inst. Trans. 37 (1946) T285-T292.

[27] S.V. Lomov, L. Gorbatikh, I. Verpoest, A model for the compression of a random assembly of carbon nanotubes, Carbon. 49 (2011) 2079-2091.

[28] A. Rawal, V. Kumar, Compressibility of highly porous network of carbon nanotubes, Appl. Phys. Lett. 103 (2013) 153103.

[29] V. Kumar, A. Rawal, Compression induced electrical response of entangled network of carbon nanomaterials, Polymer. 84 (2016) 117-120.

[30] T. Komori, K. Makishima, Numbers of fiber-to-fiber contacts in general fiber assemblies, Text. Res. J. 47 (1977) 13-17.

[31] J. Che, P. Chen, M.B. Chan-Park, High-strength carbon nanotube buckypaper composites as applied to free-standing electrodes for supercapacitors, J. Mater. Chem. A. 1 (2013) 40574066.

[32] D.A. Jack, C.S. Yeh, Z. Liang, S. Li, J.G. Park, J.C. Fielding, Electrical conductivity modeling and experimental study of densely packed SWCNT networks, Nanotechnology. 21 (2010) 195703.

[33] S. Toll, J.-A. Manson, Elastic compression of a fiber network, J. Appl. Mech. 62 (1995) $223-226$. 
[34] W.C. Young, R.G. Budynas, Roark's formulas for stress and strain, McGraw-Hill New York, 2002.

[35] R.S. Timsit, Electrical contact resistance: properties of stationary interfaces, in: Electr. Contacts 1998 Proc. Forty-Fourth IEEE Holm Conf. On, IEEE, 1998: pp. 1-19.

[36] R.B. Finch, Part II: Theory of the Pressure Distribution and Contact Area Between Fibers, Text. Res. J. 21 (1951) 383-392.

[37] A. Kukovecz, Z. Konya, N. Nagaraju, I. Willems, A. Tamasi, A. Fonseca, J.B. Nagy, I. Kiricsi, Catalytic synthesis of carbon nanotubes over $\mathrm{Co}, \mathrm{Fe}$ and $\mathrm{Ni}$ containing conventional and sol-gel silica-aluminas, Phys. Chem. Chem. Phys. 2 (2000) 3071-3076.

[38] R. Smajda, Á. Kukovecz, Z. Kónya, I. Kiricsi, Structure and gas permeability of multi-wall carbon nanotube buckypapers, Carbon. 45 (2007) 1176-1184.

[39] K.H. Kim, Y. Oh, M.F. Islam, Graphene coating makes carbon nanotube aerogels superelastic and resistant to fatigue, Nat. Nanotechnol. 7 (2012) 562-566.

[40] N. Pan, J. Chen, M. Seo, S. Backer, Micromechanics of a planar hybrid fibrous network, Text. Res. J. 67 (1997) 907-925. 